\title{
ABORDAGEM MULTICRITÉRIO HÍBRIDA WINGS-TOPSIS-PROMÉTHÉE II PARA ORDENAÇÃO DOS PAÍSES CONSUMIDORES DE GÁS NATURAL
}

\author{
Ester Figueiredo Girão \\ IBMEC-RJ \\ Av. Presidente Wilson, 118. Centro, Rio de Janeiro. CEP 20.030-020 \\ estergirao@hotmail.com \\ Luiz Flavio Autran Monteiro Gomes \\ IBMEC-RJ \\ Av. Presidente Wilson, 118, sala 1.110. Centro, Rio de Janeiro. CEP 20.030-020 \\ luiz.gomes@ibmec.edu.br
}

\begin{abstract}
RESUMO
As sociedades do mundo todo estão muito empenhadas em caminhar cada vez mais para a chamada economia de baixo carbono. Nesta nova economia, o gás natural é considerado o combustível de transição entre os mais fósseis, como a gasolina e o diesel, e os renováveis. É neste contexto atual, em que importância do gás natural na matriz energética do mundo cresce, que esta dissertação demonstra uma aplicação de uma abordagem multicritério à decisão para a ordenação dos potenciais países consumidores de gás natural do mundo. Esta ordenação tem por objetivo apoiar à decisão dos interessados no mercado de gás natural mundial fornecendo uma ordem de prioridade para o incentivo à venda deste combustível no mercado mundial. Os critérios de julgamento para ordenação são a variação de consumo de gás natural entre 2014 a 2016, volume de produção de gás natural entre 2014 e 2016 e reservas provadas de gás natural de 2016. Todos os dados utilizados que demonstram o desempenho dos países estudados foram obtidos a partir da publicação do Anuário Estatístico Brasileiro do Petróleo, Gás Natural e Biocombustíveis 2017 da ANP. A abordagem multicritério constituída nesta dissertação foi composta pelo método WINGS, utilizado para gerar os pesos de cada critério, seguido do método TOPSIS, utilizado para pré-selecionar os países que mais se aproximam de ser um potencial consumidor mundial de gás natural. Após a aplicação do método TOPSIS, foi realizada uma pré-análise de dominância entre as alternativas, a fim de excluir alternativas dominadas do conjunto de alternativas. Em seguida, foi aplicado o método PROMÉTHÉE II para estabelecer a ordenação final dos países estudados.
\end{abstract}

Palavras-chave: Gás Natural; Ordenação de Países; Apoio Multicritério à Decisão; WINGS; TOPSIS; PROMÉTHÉE II.

\begin{abstract}
Societies around the world are all very committed to moving ever more towards the so-called low carbon economy. In this new economy natural gas is regarded as the transition fuel between the most fossil fuels such as gasoline and diesel and renewables. It is in this current context, when the importance of natural gas in the world's energy matrix grows, that this paper demonstrates the application of a multi-criteria approach to the decision for the ordering of potential countries consumers of natural gas in the world. This ordering aims to support the interested parties in the world natural gas market by providing an order of priority to encourage the sale of this fuel in the world market. The judgment criteria to establish this ordering are the variation of natural gas consumption between 2014 and 2016, natural gas production volume in the same period and proven reserves of natural gas in 2016. All the data used
\end{abstract}


to demonstrate the performance of the countries studied were obtained from the publication of the ANP's 2017 Brazilian Statistical Yearbook of Oil, Natural Gas and Biofuels. The multicriteria approach developed in this paper was composed by the method WINGS, used to generate the weights of each criterion, followed by the TOPSIS method, used to preselect the countries that are closest to becoming a potential world consumer of natural gas. After the application of the TOPSIS method, we carried out a pre-analysis of dominance among alternatives. This was done in order to exclude dominated alternatives of the set of alternatives. Next, we applied the method PROMÉTHÉE II to establish the final ordering of the countries studied.

Keywords: Natural Gas; Ordering Countries; Multicriteria Decision Making; WINGS; TOPSIS; PROMÉTHÉE II.

\section{Como Citar:}

GIRÃO, Ester Figueiredo; GOMES, Luiz Flavio Autran Monteiro. Abordagem Multicritério Híbrida WINGS-TOPSIS-PROMÉTHÉE II para Ordenação dos Países Consumidores de Gás Natural. In: SIMPÓSIO DE PESQUISA OPERACIONAL E LOGÍSTICA DA MARINHA, 19., 2019, Rio de Janeiro, RJ. Anais [...]. Rio de Janeiro: Centro de Análises de Sistemas Navais, 2019.

\section{INTRODUÇÃO}

O gás natural é uma mistura de hidrocarbonetos leves que, em condições normais de temperatura e pressão, permanece no estado gasoso (SANTOS, 2002). Na natureza, o gás natural é encontrado em acumulações rochas porosas no subsolo (terrestre ou marinho), em geral acompanhado de petróleo (FARAMAWY; ZAKI; SAKR, 2016).

Segundo IEA (2017) o gás natural fornece $22 \%$ da energia utilizada mundialmente e representa quase um quarto da geração de eletricidade, além de desempenhar um papel crucial como matéria-prima para a indústria. O gás natural é um combustível versátil e seu crescimento está ligado em parte a seus benefícios ambientais em relação a outros combustíveis fósseis (MAC KINNON; BROUWER; SAMUELSEN, 2018).

O mercado de gás natural está se tornando mais globalizado. À medida que o comércio de gás aumenta, também aumentam as preocupações com a segurança do gás natural, já que um choque de demanda ou oferta em uma região pode agora ter repercussões em outras (IEA, 2018). Empresas de petróleo e gás natural têm procurado se preparar para um novo cenário em que seu modelo de negócio tradicional tem sua competitividade desafiada. As sociedades do mundo todo estão muito empenhadas em caminhar cada vez mais para a chamada economia de baixo carbono (ZHONG; BAZILIAN, 2018).

Do ponto de vista teórico, a liberalização dos mercados de combustíveis é vista por alguns por fornecer segurança energética e eficiência de alocação de recursos escassos no curto prazo (RADETZKI, 1999). No entanto, como identificadas por Bilgin (2009) e Helen (2010), condições estruturais e institucionais muitas vezes impedem a eficiência dos mercados de combustíveis e previsões de demanda confiáveis são necessárias para sustentar o verdadeiro impacto do consumo de combustível nas gerações futuras.

Diante disto, esta pesquisa buscou, através de uma abordagem multicritério híbrida, ordenar os países consumidores de gás natural no mundo. Esta ordenação teve por objetivo identificar os principais países consumidores de gás natural a fim de gerar informação relevante para o planejamento e tomada de decisões de produtores e demais envolvidos no mercado de gás natural mundial. Na abordagem multicritério aqui constituída, o conjunto de critérios foi formado pelos atributos que, segundo o Anuário Estatístico Brasileiro do Petróleo, Gás Natural e Biocombustíveis - ANP (2017) servem como referência à contextualização da indústria nacional no cenário internacional. São eles: consumo, produção 
e reservas de gás natural de cada país estudado no anuário. O conjunto de alternativas foi formado pelos países estudados neste anuário que possuem informações registradas nestes 3 critérios definidos.

Na aplicação da abordagem, foram aplicados 3 métodos em sequência. O primeiro deles foi o método WINGS. Este método foi utilizado para estabelecer o peso de cada critério. Após isto, foi aplicado o método TOPSIS ao conjunto de alternativas para pré-selecionar os 15 principais países consumidores de gás natural no mundo. Em seguida, foi realizada uma pré-análise de dominância entre os 15 países pré-selecionados pelo método TOPSIS. Por fim, aplicou-se o método PROMÉTHÉE II ao conjunto de alternativas constituído após a préanálise de dominância.

A importância da indústria de petróleo e gás natural para a sociedade global justifica a realização do estudo aqui apresentado, visto que estes combustíveis são as fontes energéticas mais utilizadas atualmente. Neste âmbito, a busca pelo padrão de excelência em planejamento e gestão, aliada ao investimento no aumento da capacidade para atender as demandas são importantes para o desenvolvimento da indústria de petróleo e gás natural e induzem decisões e análises que abrangem múltiplos critérios, além de diferentes grupos de stakeholders.

\section{REFERENCIAL TEÓRICO}

\subsection{O GÁS NATURAL: COMPOSIÇÃO, CADEIA DE VALOR E PARTICIPAÇÃO NA MATRIZ ENERGÉTICA}

A composição do gás natural pode variar de campo para campo. Como principais fatores desta variação podem-se destacar o tipo de matéria orgânica que lhe deu origem, os processos naturais a que foi submetido, estar associado ou não ao óleo e ter sido ou não processado em unidades industriais. No entanto, o gás natural compõe-se, principalmente, de metano, etano, propano e, em menores proporções, de outros hidrocarbonetos de metano $\left(\mathrm{CH}_{4}\right)$. Normalmente, o gás natural apresenta baixos teores dē impurezas como nitrogênio $\left(\mathrm{N}_{2}\right)$, dióxido de carbono $\left(\mathrm{CO}_{2}\right)$, água e compostos de enxofre.

Conceitualmente, a cadeia de valor do gás natural segue uma estrutura semelhante à da cadeia do petróleo, sendo igualmente dividida em três segmentos: upstream, midstream e downstream.

Com relação ao segmento upstream, a exploração é o processo de pesquisa de acumulação de hidrocarbonetos, tanto em bacias terrestres (onshore) como em bacias marítimas (offshore). A produção é o processo de extração, recuperação e processamento do gás natural em escala comercial. A fase de exploração é tipicamente caracterizada por alto grau de risco, principalmente ligado à incerteza da descoberta de uma jazida em áreas com conhecimento geológico ainda limitado e, portanto, com elevados investimentos e custos operacionais.

No que se refere ao midstream, a escolha da forma de movimentação do gás natural entre a jazida produtora e o mercado consumidor é uma questão estratégica para a indústria do gás natural.

Por último, no downstream, após a etapa de transporte, inicia-se o processo de distribuição, a partir do recebimento do gás nos chamados citygates, que são instalações de redução e controle de pressão, medição e odorização do gás natural. A partir dos citygates, o gás natural é direcionado através de tubulações aos diversos segmentos do mercado: industrial, comercial, residencial e geração de eletricidade.

Diante do notável potencial impacto do gás natural em diversos setores e na geopolítica mundial percebe-se como prioritário fomentar a geração de estratégias de desenvolvimento energético mundial contemplando projetos de exploração e produção, processamento e transporte de gás natural com o objetivo de aumentar a sua oferta. 


\subsection{MÉTODOS DE APOIO MULTICRITÉRIO À DECISÃO}

Os métodos de apoio multicritério à decisão contribuem na obtenção de elementos de resposta às questões de um agente de decisão no decorrer de um processo decisório normalmente considerado complexo. Esses elementos têm como objetivo esclarecer cada decisão e recomendá-la. Uma metodologia de apoio multicritério à decisão procura fazer com que o processo seja o mais neutro, objetivo, válido e transparente possível, sem predefinir ao decisor uma solução única e verdadeira (GOMES; ARAYA; CARIGNANO, 2004). Assim, os métodos de apoio multicritério à decisão aplicam-se em inúmeras áreas sempre que se deseje selecionar, ordenar, classificar ou descrever alternativas presentes em um processo decisório na presença de múltiplos critérios - quantitativos e qualitativos (ROY; BOUYSSOU, 1993; ROMERO, 1993; VINCKE, 1989). Estes métodos buscam auxiliar um processo decisório em que um conjunto de alternativas deve ser analisado simultaneamente por um conjunto de critérios que são, quase sempre, conflitantes (GOMES; ARAYA; CARIGNANO, 2004).

Muitas classificações são utilizadas para denominar os métodos multicritérios de apoio à decisão. Uma destas classificações é a que subdivide os métodos da Escola Americana e os métodos da Escola Francesa, também denominada Escola Europeia. Existem outros métodos multicritério que, no entanto, não se enquadram formalmente dentro de uma dessas duas escolas, chamados métodos híbridos (GOMES; GOMES; ALMELDA, 2006; BELTON; STEWART, 2002; BARBA- ROMERO; POMEROL, 1997). Como exemplos de métodos híbridos, podem-se citar o método TODIM (GOMES; RANGEL, 2009; GOMES; ARAYA; CARIGÑANO, 2004), o método MACBETH (BANA e COSTA; CORTE; VANSNICK, 2005) e o método TOPSIS (HWANG; YOON, 1981).

Os principais métodos da Escola Francesa são os métodos ELECTRE (ROY; BOUYSSOU, 1993) e os métodos PROMÉTHÉE (BRANS; VINCKE; MARESCHAL, 1986). Como principais métodos da Escola Norte-Americana, pode-se citar o método AHP (Analytic Hierarchy Process) (SAATY, 1980) e a Teoria da Utilidade Multiatributo (Multiattribute Utility Theory) (KEENEY; RAIFFA, 1993).

\subsection{MÉTODOS MULTICRITÉRIO DE APOIO À DECISÃO APLICADOS NA INDÚSTRIA DE ÓLEO E GÁS}

De acordo com Neves, Pereira e Costa (2015) a utilização de métodos de apoio multicritério à tomada de decisão no planejamento e gestão da indústria de petróleo e gás natural tem evoluído ao longo dos anos, mas é ainda pouca explorada em temas como: gás natural, downstream e offshore. Estes autores ressaltam que os primeiros artigos acerca deste tema foram publicados em 1996. Com referência aos descritores utilizados na pesquisa (fonte energética, segmento da cadeia produtiva e posicionamento geográfico), conclui-se que dos 48 trabalhos selecionados da base Scopus ou da base ISI Web of Knowledge 41,67\% referiamse a petróleo, 35,42 \% a petróleo e gás natural e 22,92\% a gás natural.

Ainda sobre o artigo de Neves, Pereira e Costa (2015), é interessante notar que o método AHP foi o mais utilizado, no contexto dos artigos analisados, para planejamento e gestão na indústria de petróleo e gás, representando 60,94\% das ocorrências nesses artigos. A análise do artigo também permite observar que 1,56\% dos trabalhos adotou o método PROMÉTHÉE II e 3,13\% o TOPSIS.

No artigo de Gomes e Maranhão (2008), deseja-se conhecer qual a melhor opção em um conjunto de alternativas para destinação das reservas de gás natural descobertas recentemente na Bacia de Santos, mais especificamente no campo de Mexilhão. A análise multicritério empregada no estudo fez uso do método TODIM, método multicritério discreto fundamentado na Teoria dos Prospectos. A aplicação do método TODIM mostrou-se bastante útil na recomendação de opções de projetos no upstream, devido ao fato de permitir a clara identificação de alternativas mais importantes, em face aos cenários testados e em relação aos 
critérios explicitados.

De acordo com Khosravanian e Wood (2016) as empresas de petróleo e gás natural tomam várias decisões para a seleção de um projeto adequado de completação de poço que consiste em equipá-lo para a produção de óleo ou gás ou para injeção de fluidos no reservatório. Selecionar alternativas de projetos de completação visa obter uma maior produtividade do poço, menores investimentos, menores custos de manutenção e menor tempo para a sua conclusão. A decisão geralmente se torna estratégica; impondo prioridades do operador sobre as hipóteses de desempenho e investimento definidas para cada alternativa.

\section{METODOLOGIA}

Conforme descrito na Introdução, o principal objetivo deste artigo foi gerar informação relevante às informações divulgadas no Anuário Estatístico Brasileiro do Petróleo, Gás Natural e Biocombustíveis - ANP (2017) que serviu como guia para planejamento e tomada de decisão dos stakeholders deste mercado. Então, foi estabelecida uma ordenação para o conjunto dos potenciais países consumidores de gás natural no mundo. Para isto, foi desenvolvida uma abordagem multicritério composta em sequência pelos métodos WINGS, TOPSIS e PROMÉTHÉE II.

\subsection{SELEÇÃO DE CONJUNTO DE ALTERNATIVAS}

O conjunto de alternativas foi composto pelos 39 países consumidores de gás natural no mundo que apresentam dados de consumo, produção e reserva (ANP, 2017) conforme Tabela 1.

Tabela 1 - Conjunto de alternativas

\begin{tabular}{|r|c|c|c|c|c|}
\hline \multicolumn{7}{|c|}{ Alternativas } \\
\hline $\mathbf{1}$ & Canadá & $\mathbf{1 4}$ & Holanda & $\mathbf{2 7}$ & Emirados Árabes Unidos \\
\hline $\mathbf{2}$ & Estados Unidos & $\mathbf{1 5}$ & Itália & $\mathbf{2 8}$ & Irã \\
\hline $\mathbf{3}$ & México & $\mathbf{1 6}$ & Noruega & $\mathbf{2 9}$ & Argélia \\
\hline $\mathbf{4}$ & Argentina & $\mathbf{1 7}$ & Polônia & 30 & Egito \\
\hline $\mathbf{5}$ & Brasil & $\mathbf{1 8}$ & Reino Unido & 31 & Austrália \\
\hline $\mathbf{6}$ & Colômbia & $\mathbf{1 9}$ & Romênia & 32 & Bangladesh \\
\hline $\mathbf{7}$ & Peru & $\mathbf{2 0}$ & Rússia & 33 & China \\
\hline $\mathbf{8}$ & Trinidad e Tobago & $\mathbf{2 1}$ & Turcomenistão & 34 & Índia \\
\hline $\mathbf{9}$ & Venezuela & $\mathbf{2 2}$ & Ucrânia & 35 & Indonésia \\
\hline $\mathbf{1 0}$ & Alemanha & $\mathbf{2 3}$ & Uzbequistão & 36 & Malásia \\
\hline $\mathbf{1 1}$ & Azerbaijão & $\mathbf{2 4}$ & Arábia Saudita & 37 & Paquistão \\
\hline $\mathbf{1 2}$ & Cazaquistão & $\mathbf{2 5}$ & Catar & 38 & Tailândia \\
\hline $\mathbf{1 3}$ & Dinamarca & $\mathbf{2 6}$ & Coveite & 39 & Vietnã \\
\hline
\end{tabular}

Fonte: Elaborado pelos autores.

\subsection{SELEÇÃO DO CONJUNTO DE CRITÉRIOS E SUBCRITÉRIOS}

O conjunto de critérios e subcritérios foi estabelecido conforme demonstrado na Tabela 2. 
Tabela 2 - Conjunto de critérios e subcritérios

\begin{tabular}{|c|c|c|c|c|}
\hline \multicolumn{2}{|c|}{ Critérios } & $\begin{array}{l}\text { Volume de consumo de gás } \\
\text { natural em bilhões de }{ }^{3}\end{array}$ & $\begin{array}{c}\text { Volume de produção de gás } \\
\text { natural em bilhões de } \mathrm{m}^{3}\end{array}$ & $\begin{array}{c}\text { Volume de reservas de } \\
\text { gás natural em trilhões de } \\
\text { m }^{3} \text { em } 2016 .\end{array}$ \\
\hline \multirow{3}{*}{ Subcritérios } & 1 & $\begin{array}{l}\text { Variação percentual do con- } \\
\text { sumo de gás natural no perí- } \\
\text { odo de } 2013 \text { a } 2014\end{array}$ & $\begin{array}{l}\text { Produção de gás natural } \\
\text { em bilhões de } \mathrm{m}^{3} \text { em } \\
2014\end{array}$ & \multirow{3}{*}{ Não há subcritérios } \\
\hline & 2 & $\begin{array}{l}\text { Variação percentual do con- } \\
\text { sumo de gás natural no perí- } \\
\text { odo de } 2014 \text { a } 2015\end{array}$ & $\begin{array}{l}\text { Produção de gás natural } \\
\text { em bilhões de } \mathrm{m}^{3} \text { em } \\
2015\end{array}$ & \\
\hline & 3 & $\begin{array}{l}\text { Variação percentual do con- } \\
\text { sumo de gás natural no perí- } \\
\text { odo de } 2015 \text { a } 2016\end{array}$ & $\begin{array}{l}\text { Produção de gás natural } \\
\text { em bilhões de } \mathrm{m}^{3} \text { em } \\
2016\end{array}$ & \\
\hline
\end{tabular}

\subsection{ABORDAGEM MULTICRITÉRIO WINGS-TOPSIS-PROMÉTHÉE II}

Definidos o conjunto de alternativas e conjunto de critérios, descreve-se a abordagem multicritério que foi empregada.

\subsubsection{Aplicação do método WINGS para estabelecer peso dos critérios}

O método WINGS, segundo Michnik (2013) foi escolhido para gerar os pesos de cada critério porque considera em sua metodologia a força de cada critério para a venda de gás natural, ou seja, o grau de força de cada critério no conjunto de critérios, e também o grau de influência que os critérios exercem entre si.

Pela natureza dos critérios estudados, pode-se perceber que eles influenciam um ao outro. Por exemplo: um país que possua boas reservas de gás natural pode influenciar sua produção e um país com alta produção de gás natural pode influenciar seu consumo. Além disso, cada um dos critérios estudados possui uma força para tornar cada país um potencial consumidor. Por exemplo: o consumo possui uma força maior para tornar determinado país potencial consumidor de gás natural do que seu volume de reservas.

Para aplicar o método WINGS e, assim, gerar o peso de cada critério, foi construída a matriz D força-influência (Tabela 3) com valores que variam de 0 a 4 de acordo com a seguinte escala: (0) sem força; (1) força baixa; (2) força moderada; (3) força alta; e (4) força muito alta; para avaliar o grau de força de cada critério no sistema em estudo. E para avaliar a influência de cada critério sobre os demais de acordo com a seguinte escala: (0) sem influência; (1) pouca influência; (2) influência moderada; (3) influência alta; (4) influência muito alta.

A referência de força (importância) e de influência para cada critério foram avaliadas pelos autores com base em estudos realizados anteriormente e suas experiências profissionais no segmento da indústria de petróleo. A referência de força expressa quanto o critério pode ser capaz de impactar a venda de gás natural no mundo. E a referência de influência expressa o quanto um critério influencia outro critério na venda de gás natural.

O primeiro critério avaliado foi consumo. Para o critério consumo, atribuiu-se grau de força muito alta (4), uma vez que o consumo de gás natural em uma determinada região impacta fortemente a sua venda. 
O segundo critério avaliado foi produção. Para o critério produção, atribuiu-se grau de força moderada (2), uma vez que a produção de gás natural em uma determinada região pode impactar a venda. Se determinada região já possui produção de gás natural, isso pode impactar a venda positivamente ou não. Porém, não com a mesma intensidade que a variação do consumo pode contribuir.

O terceiro critério avaliado foi reserva. Para o critério reserva, atribuiu-se grau de força fraca (1), porque o fato de uma determinada localidade possuir reservas de gás natural não representa alto impacto nas vendas, mas pode influenciar de alguma forma.

Tendo composto o grau de força de cada componente no sistema, atribuiu-se o grau de influência entre os componentes. Foi estabelecido grau de influência (3) do componente consumo sobre a componente produção. Isto porque, o consumo pode influenciar de forma alta a produção de gás natural. Foi estabelecido grau de influência (0) do consumo sobre reserva, pois não é possível o consumo influenciar o nível de reservas de gás natural por se tratar de um bem natural.

Atribuiu-se: grau de influência (2) da produção sobre o consumo porque a produção de gás natural pode influenciar seu consumo de forma moderada, pois pode-se produzir e não consumir; grau de influência (0) da produção sobre reserva porque a produção de gás natural não pode influenciar o volume de reservas.

Atribuiu-se: grau de influência (1) da reserva sobre o consumo porque o volume de reserva de gás natural pode influenciar seu o consumo de forma baixa; grau de influência 3 da reserva sobre a produção, porque possuir reserva de gás natural influencia fortemente sua produção. Porém, ainda existe a possibilidade de possuir reservas e não produzir gás natural.

Tabela 3 - Matriz D força - influência dos critérios para aplicação do método WINGS

\begin{tabular}{|l|c|c|c|}
\cline { 2 - 4 } \multicolumn{1}{c|}{} & \multicolumn{3}{c|}{ Matriz D } \\
\hline Critério & Consumo & Produção & Reserva \\
\hline Consumo & 4 & 3 & 0 \\
\hline Produção & 2 & 2 & 0 \\
\hline Reserva & 1 & 3 & 1 \\
\hline
\end{tabular}

Fonte: Elaborado pelos autores.

Após a aplicação do método WINGS, o valor do efeito líquido foi utilizado $(r-c)$ para designar o peso de cada critério. Isto acontece, porque o efeito líquido é a diferença do impacto exercido com o impacto recebido por cada critério no conjunto de critérios. Como o valor do efeito líquido pode ser positivo ou negativo, seus valores foram elevados ao quadrado para eliminar os valores negativos. Em seguida, foi extraída a raiz quadrada dos valores do efeito líquido elevados ao quadrado. Isto aconteceu para se obter todos os valores do efeito líquido positivos e, então, designar os pesos dos critérios, calculando um novo efeito líquido (NEL) (Equação 1).

$$
N E L=\sqrt{\left(r_{i}-c_{i}\right)^{2}}
$$

Após obter os valores extraídos da Equação 1, estes valores foram normalizados de acordo com a Equação 2 para obter o peso de cada critério.

$$
w_{i}=\frac{\sqrt{\left(r_{i}-c_{i}\right)^{2}}}{\sum_{i=1}^{n} \sqrt{\left(r_{i}-c_{i}\right)^{2}}}
$$

Na Equação $2 w_{i}$ é o peso do critério $i$.

\subsubsection{Estabelecer peso aos critérios e subcritérios}

Conforme explicado na Seção 3.2, como foi utilizada a variação do consumo no período de 2014 a 2016, cada ano representa um subcritério do critério Consumo. E, como foi utilizado o valor da produção no mesmo período, cada ano representa um subcritério do critério Produção. O critério Reserva não possui subcritérios.

Para estabelecer o peso de cada subcritério do critério Consumo foi calculado o 
desvio-padrão da variação de consumo dos países estudados em cada ano, conforme Equação 3.

$$
S_{s c_{i}}=\sqrt{\frac{\sum\left(x_{i}-\bar{x}\right)^{2}}{n-1}}
$$

Na Equação $3 \mathrm{~S}_{\text {sci }}$ é o desvio-padrão do subcritério $i$. $\mathrm{x}_{i}$ é um dado do subcritério $i$ e $\overline{\mathrm{x}}$ é a média dos dados do subcritério $i$. Cada peso foi dado proporcionalmente ao valor do respectivo desvio-padrão. Então, valores dos subcritérios que pertencem ao mesmo critério foram normalizados, criando a variável $u$ (Equação 4).

$$
u_{i}=\frac{S_{s c_{i}}}{\sum_{i=1}^{n} S_{s c_{i}}}
$$

Após isto, o valor do desvio-padrão normalizado de cada subcritério $(u)$ foi multiplicado pelo peso de cada critério já designado anteriormente pelo método WINGS para obter o peso de cada subcritério (Equação 5). O mesmo foi feito para atribuir os pesos dos subcritérios do critério Produção. Como o critério Reservas não possui subcritérios foi atribuído a ele o valor gerado pelo método WINGS.

$$
w_{s c_{i}}=w_{i} * u_{i}
$$

Na Equação $5 w_{s c_{i}}$ é o peso do subcritério $i$.

Assim, o critério que obteve maior desvio-padrão foi o que possui maior peso. Isto porque neste critério existem alternativas que alcançaram um desempenho superior à média, enquanto outras alcançaram desempenho inferior à média. Desta forma, foi refletida na análise de forma favorável (positiva) as alternativas que possuíram desempenho superior à média enquanto outras possuíram desempenho inferior. Da mesma forma foi refletida na análise de forma desfavorável (negativa) as alternativas que possuíram desempenho inferior à média enquanto outras possuíram desempenho superior.

O critério que obteve menor desvio-padrão deve possuir menor peso. Isto porque, neste critério o desempenho das alternativas encontra-se mais próximo da média em relação aos outros critérios. Então, deve-se representar de forma menos gradual os desvios dos desempenhos das alternativas em relação à média.

Após se realizar os cálculos do método WINGS, os valores do efeito líquido (r-c) foram elevados ao quadrado para eliminar números negativos. Em seguida, foi extraída a raiz quadrada destes valores. Após isto, normalizaram-se os valores dos resultados da raiz quadrada a fim de estabelecer o peso de cada critério seguindo as Equações 1 e 2. (Tabela 4).

Tabela 4 - Peso dos critérios

\begin{tabular}{|l|c|c|}
\hline \multicolumn{1}{|c|}{ Critério } & NEL & Wi \\
\hline Consumo & 0,0329 & 0,0803 \\
\hline Produção & 0,0535 & 0,1305 \\
\hline Reserva & 0,3235 & 0,7892 \\
\hline \multicolumn{2}{|c|}{ Fonte: Elaborado pelos autores. }
\end{tabular}

\subsubsection{Aplicação do método TOPSIS: pré-seleção de alternativas}

Nesta etapa da metodologia foi estabelecida uma pré-seleção de alternativas para encontrar os 15 principais países consumidores de gás natural dentro do universo de 39 países divulgados no Anuário da ANP de 2017 a fim de reduzir o número de alternativas (Tabela 5). Nesta aplicação foram analisados os desempenhos do conjunto de todas as alternativas no conjunto de critérios descrito na Seção 3.2. Os subcritérios $\mathrm{SC}_{1}, \mathrm{SC}_{2}$ e $\mathrm{SC}_{3}$ são subcritérios de benefício, e os demais subcritérios $\mathrm{SC}_{4}, \mathrm{SC}_{5}, \mathrm{SC}_{6}$ e $\mathrm{SC}_{7}$ são de custo.

O método multicritério híbrido TOPSIS foi escolhido para estabelecer a pré-seleção de alternativas porque não realiza uma comparação par a par entre estas (HWANG; YOON, 
1981). Este método estabelece uma ordenação para o conjunto de alternativas a partir da alternativa que mais se aproxima da solução ideal positiva e que está mais distante da solução ideal negativa.

Desta forma, a alternativa que possuiu o maior crescimento do consumo de gás natural, a menor produção e as menores reservas foi a alternativa que possuiu o melhor desempenho em todos os critérios.

Tabela 5 - Ordenação dos 15 primeiros países pré-selecionados

\begin{tabular}{|c|c|c|}
\hline Ordenação & Alternativa & $\boldsymbol{\xi}$ \\
\hline $1^{\text {o }}$ & Bangladesh & 0,9789 \\
\hline $2^{\text {o }}$ & Peru & 0,9783 \\
\hline $3^{\text {o }}$ & México & 0,9684 \\
\hline $4^{\text {o }}$ & Argentina & 0,9677 \\
\hline $5^{\text {o }}$ & Colômbia & 0,9675 \\
\hline $6^{\text {o }}$ & Taliândia & 0,9674 \\
\hline $7^{\circ}$ & Polônia & 0,9671 \\
\hline $8^{\text {o }}$ & Vietnã & 0,9669 \\
\hline $9^{\circ}$ & Paquistão & 0,9666 \\
\hline $10^{\circ}$ & Cazaquistão & 0,9630 \\
\hline $11^{\circ}$ & Itália & 0,9630 \\
\hline $12^{\circ}$ & Reino Unido & 0,9626 \\
\hline $13^{\circ}$ & Brasil & 0,9612 \\
\hline $14^{\circ}$ & Azerbaijão & 0,9601 \\
\hline $15^{\circ}$ & Alemanha & 0,9600 \\
\hline \multicolumn{3}{|c|}{ Fonte: Elaborado pelos autores. } \\
\hline
\end{tabular}

\subsubsection{Pré-análise de dominância entre as alternativas}

A pré-análise de dominância é um método de comparação que simplifica o problema de decisão pela eliminação de alternativas dominadas em relação às demais. A eliminação é realizada pela comparação e seleção da alternativa na qual para todos os atributos existe uma alternativa tão boa quanto à dominada e para pelo menos um atributo a dominada é pior (POMEROL; BARBA-ROMERO, 2000; LEÃO, 2007).

Nesta etapa da abordagem WINGS-TOPSIS-PROMÉTHÉE II verificou-se se existe alguma alternativa dominada no conjunto das 15 alternativas pré-selecionadas pelo método TOPSIS na etapa anterior (Tabela 5).

Então, realizou-se a comparação entre os atributos de todas as alternativas e, em seguida, foram excluídas da análise as alternativas nas quais para todos os atributos existia uma alternativa tão boa quanto a dominada e para pelo menos um atributo a dominada é pior. Os subcritérios de variação de consumo $\left(\mathrm{SC}_{1}, \mathrm{SC}_{2}\right.$ e $\left.\mathrm{SC}_{3}\right)$ são de maximização, os subcritérios de produção $\left(\mathrm{SC}_{4}, \mathrm{SC}_{5}\right.$ e $\left.\mathrm{SC}_{6}\right)$ e de reservas provadas $\left(\mathrm{SC}_{7}\right)$ são de minimização.

Após a pré-análise de dominância entre os 15 países selecionados inicialmente, a partir do método TOPSIS, foi obtida a ordenação final do conjunto dos 12 primeiros países que foram objeto de estudo para aplicação do método PROMÉTHÉE II conforme Tabela 6.

Tabela 6 - Ordenação final dos 12 primeiros países selecionados

\begin{tabular}{|c|c|c|}
\hline Ordenação & Alternativa & $\boldsymbol{\xi}$ \\
\hline $1^{\text {o }}$ & Bangladesh & 0,9789 \\
\hline $2^{\text {o }}$ & Peru & 0,9783 \\
\hline $3^{\text {o }}$ & México & 0,9684 \\
\hline $4^{\circ}$ & Argentina & 0,9677 \\
\hline $5^{\circ}$ & Colômbia & 0,9675 \\
\hline $6^{\circ}$ & Polônia & 0,9671 \\
\hline $7^{\circ}$ & Vietnã & 0,9669 \\
\hline $8^{\text {o }}$ & Itália & 0,9630 \\
\hline $9^{\circ}$ & Reino Unido & 0,9626 \\
\hline $10^{\circ}$ & Brasil & 0,9612 \\
\hline
\end{tabular}




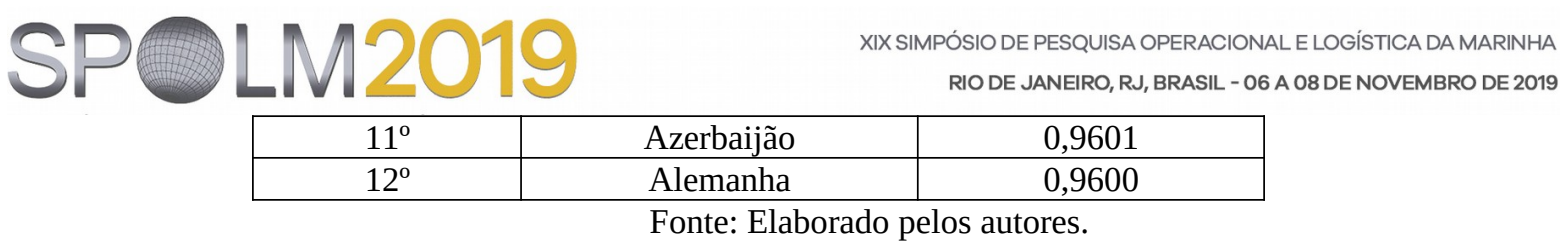

\subsubsection{Aplicação do método PROMÉTHÉE II}

Nesta etapa da abordagem foi aplicado o método PROMÉTHÉE II para estabelecer a ordenação final do novo conjunto de alternativas, isto é, o conjunto de alternativas resultante da pré-seleção estabelecida pelo método TOPSIS e pela exclusão das alternativas dominadas.

O PROMÉTHÉE II foi escolhido porque deseja-se aplicar um método que realize a comparação entre pares de alternativas em todos os critérios.

Para a aplicação do método PROMÉTHÉE II é necessário designar uma função de preferência para cada critério. Isto é, a forma pela qual a diferença de desempenho de todas as alternativas é medida no intervalo de 0 até 1 . Nesta metodologia, foi utilizado o Critério de Preferência Linear para medir a diferença de desempenho entre as alternativas em todos os critérios. Isto foi adotado porque esta função de preferência mede as diferenças entre as alternativas através de todos os valores entre 0 e 1 utilizando apenas um limiar de preferência ou utilizando o Critério de Preferência Linear com Zona de Indiferença sempre com limiar de indiferença igual a zero (MLADINEO; JAJAC; ROGULJ, 2016).

Conforme demonstrado no artigo de Mladineo, Jajac e Rogulj (2016) para calcular o limiar de preferência do Critério de Preferência Linear, deve-se subtrair o valor do maior desempenho de cada critério pelo valor do pior desempenho de cada critério (Equação 5).

$$
p_{i}=x_{i}^{+}-x_{i}^{-}
$$

Na Equação $5 p_{i}$ é o limiar de preferência do critério $i$; $x_{i}^{+}$é o valor do maior desempenho no critério $i$ e $x_{i}^{-}$é o valor do menor desempenho no critério $i$.

Aṕ̣s definirem-se os limiares de preferência, o método PROMÉTHÉE II foi aplicado no conjunto de alternativas estudado utilizando estes valores como limiares e os pesos dos critérios estabelecidos pelo método WINGS.

Outros métodos multicritério também utilizam uma comparação entre pares de alternativas com o objetivo de ordenar um conjunto de alternativas, como por exemplo os métodos AHP, ANP, MACBETH, TODIM, ELECTRE II, ELECTRE III e ELECTRE IV.

Porém, os métodos AHP, ANP e MACBETH realizam uma comparação entre pares de alternativas diferente dos outros métodos citados acima. Isto acontece, porque ao compararem duas alternativas $a$ e $b$ em determinado critério, o valor resultante da comparação de $a$ para $b$ deve ser o inverso do valor de $b$ e $a$ ou vice e versa.

O método TODIM mede as diferenças entre pares de alternativas nos critérios estudados de forma diferente quando a diferença é positiva e negativa. Por causa disto, este método não deve ser utilizado nesta pesquisa, pois deseja-se medir de forma idêntica diferenças positivas e negativas.

O método ELECTRE IV trabalha com critérios que possuam pesos iguais. No cálculo dos métodos ELECTRE II e ELECTRE III a diferença entre o desempenho das alternativas nos critérios é medida pelo valor do peso de cada critério ao gerar o índice de concordância e, apenas, o valor da maior diferença entre o desempenho das alternativas nos critérios estudados para gerar o índice de discordância.

Desta forma, justifica-se o uso do método PROMÉTHÉE II porque realiza as diferenças entre desempenhos de alternativas em todos os critérios. Isto é importante para a análise dos envolvidos no mercado de gás natural sobre os dados do Anuário para considerar o valor das diferenças entre pares de alternativas em todos os critérios. Além disso, o PROMÉTHÉE II mede as diferenças dos desempenhos de forma idêntica. 


\section{ANÁLISE DOS DADOS}

\subsection{GRAu de Atratividade dos países}

Além da ordenação de alternativas, também foi possível atribuir um grau de atratividade a cada alternativa, segundo o valor de seu fluxo de superação líquido (Tabela 7).

Conforme Miettinen (2014), o objetivo da visualização gráfica em problemas multicritério é auxiliar na melhor compreensão sobre os dados. Desta forma, o decisor obtém maior conhecimento sobre os insights do problema para efetuar uma escolha mais adequada. Tendo dito isto, o gráfico PROMETHEE Rankings gerado pelo software Visual PROMETHEE ${ }^{\circledR}$ tem por objetivo oferecer informações complementares.

O gráfico PROMETHEE Rankings gerado pelo software Visual PROMETHEE® divide os valores possíveis do valor do fluxo de superação líquido em 4 intervalos iguais. Ou seja, pode-se verificar que o intervalo $(-1 ;+1)$ é dividido em 4 níveis de classificação contendo um intervalo de 0,5 cada um. A seguir na Tabela 7 apresenta-se a classificação de cada intervalo de fluxo de superação líquido de I até $\mathrm{V}$.

Tabela 7 - Níveis de graus de atratividade

\begin{tabular}{|c|c|}
\hline Fluxo de superação líquido (phi) & Grau de atratividade \\
\hline$[0,5<$ phi $\leq 1]$ & I \\
\hline$[0<p h i \leq 0,5]$ & II \\
\hline$[-0,5<p h i \leq 0]$ & III \\
\hline$[-1<$ phi $\leq-0,5]$ & IV \\
\hline$[p h i=-1]$ & $\mathrm{V}$ \\
\hline
\end{tabular}

Observando a Tabela 7, foi possível verificar que o grau de atratividade I é atribuído no caso de algum país obter fluxo de superação líquido entre o intervalo $(+0,5 ;+1)$. As demais classificações foram atribuídas em intervalos de 0,5 entre os valores do maior e menor fluxo de superação líquido possíveis na aplicação do método PROMÉTHÉE II. A Figura 1 apresenta o grau de atratividade atribuído a cada país estudado.

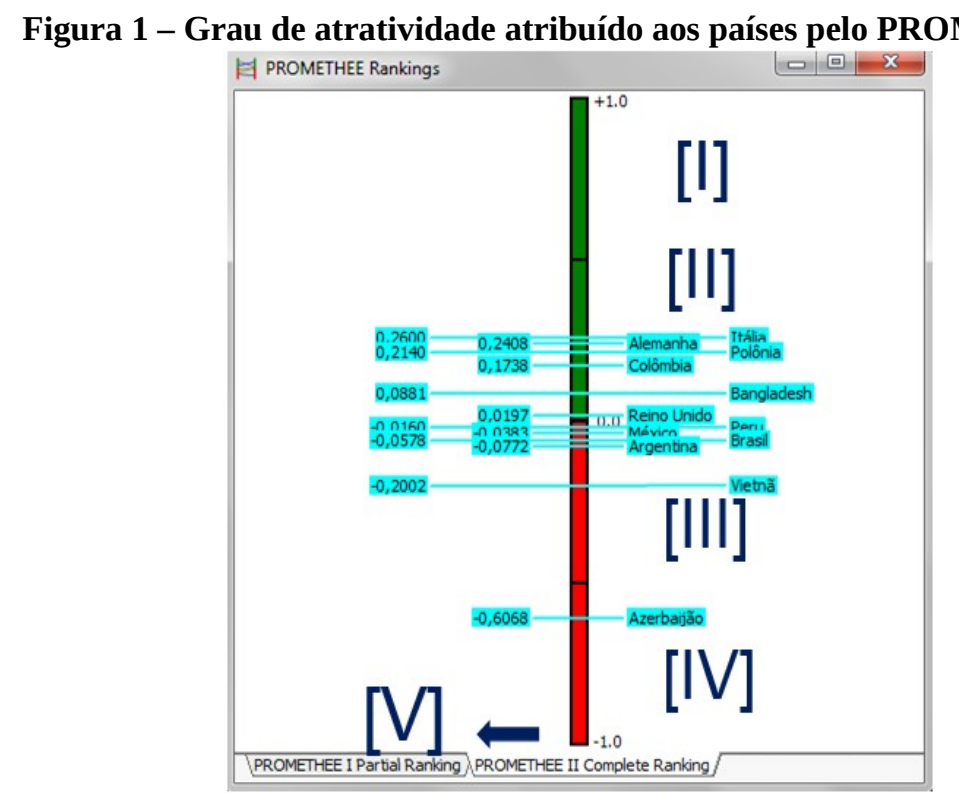

Fonte: Editado de Software Visual PROMETHEE®

Na Figura 1 observa-se que os países se encontram entre as classificações II e IV com valores de desempenho relativamente próximos. Para auxiliar a identificação dos graus de atratividade de cada país, a Tabela 8 apresenta a ordenação, os valores do fluxo de superação líquido e o grau de atratividade de cada país. 
Tabela 8 - Ordenação e grau de atratividade dos países

\begin{tabular}{|c|c|c|c|}
\hline Ordem & País & Fluxo de Superação Líquido & Grau de Atratividade \\
\hline $1^{\circ}$ & Itália & 0,2600 & II \\
\hline $2^{\circ}$ & Alemanha & 0,2408 & II \\
\hline $3^{\circ}$ & Polônia & 0,2140 & II \\
\hline $4^{\circ}$ & Colômbia & 0,1738 & II \\
\hline $5^{\circ}$ & Bangladesh & 0,0881 & II \\
\hline $6^{\circ}$ & Reino Unido & 0,0197 & II \\
\hline $7^{\circ}$ & Peru & $-0,0160$ & III \\
\hline $8^{\circ}$ & México & $-0,0383$ & III \\
\hline $9^{\circ}$ & Brasil & $-0,0578$ & III \\
\hline $10^{\circ}$ & Argentina & $-0,0772$ & III \\
\hline $11^{\circ}$ & Vietnã & $-0,2002$ & III \\
\hline $12^{\circ}$ & Azerbaijão & $-0,6068$ & IV \\
\hline
\end{tabular}

Fonte: Elaborado pelos autores.

Observando a Tabela 8, foi possível identificar que todos os países possuem grau de atratividade II, III ou IV. Nenhum país apresentou o melhor desempenho (I) nem o pior desempenho em todos os critérios $(\mathrm{V})$. $\mathrm{O}$ fato de nenhum país possuir grau de atratividade $\mathrm{V}$ significa que nenhum país possui o pior desempenho em todos os critérios.

A avaliação de grau de atratividade de cada país forneceu informações complementares importantes acerca do desempenho de cada país. Por exemplo, pode-se notar que a Itália, apesar de apresentar o primeiro lugar na ordenação, não possui grau de atratividade I (Tabela 8).

Também foi possível notar que o Brasil apesar de ocupar o $9^{\circ}$ lugar na ordenação, possui o mesmo grau de atratividade de outros países que se encontram em posição superior. Isto demonstrou que, apesar do Brasil ocupar o $9^{\circ}$ lugar na ordenação, não se encontra tão distante de outros países que se encontram em posições acima da sua e nem possui menor grau de atratividade que estes.

\subsection{PONTOS FORTES E FRACOS DE CADA PAÍS}

Nesta etapa foram analisados, separadamente, a partir do gráfico PROMETHEE Rainbow gerado pelo software Visual PROMETHEE® os pontos fortes e fracos de cada país.

A partir do gráfico PROMETHEE Rainbow foi possível realizar uma análise comparativa do desempenho dos subcritérios para cada país. Ou seja, foi possível de forma visual identificar em cada país os subcritérios nos quais estes obtiveram melhor e pior desempenhos em relação aos demais países.

Figura 2 - Pontos fortes e fracos de cada país

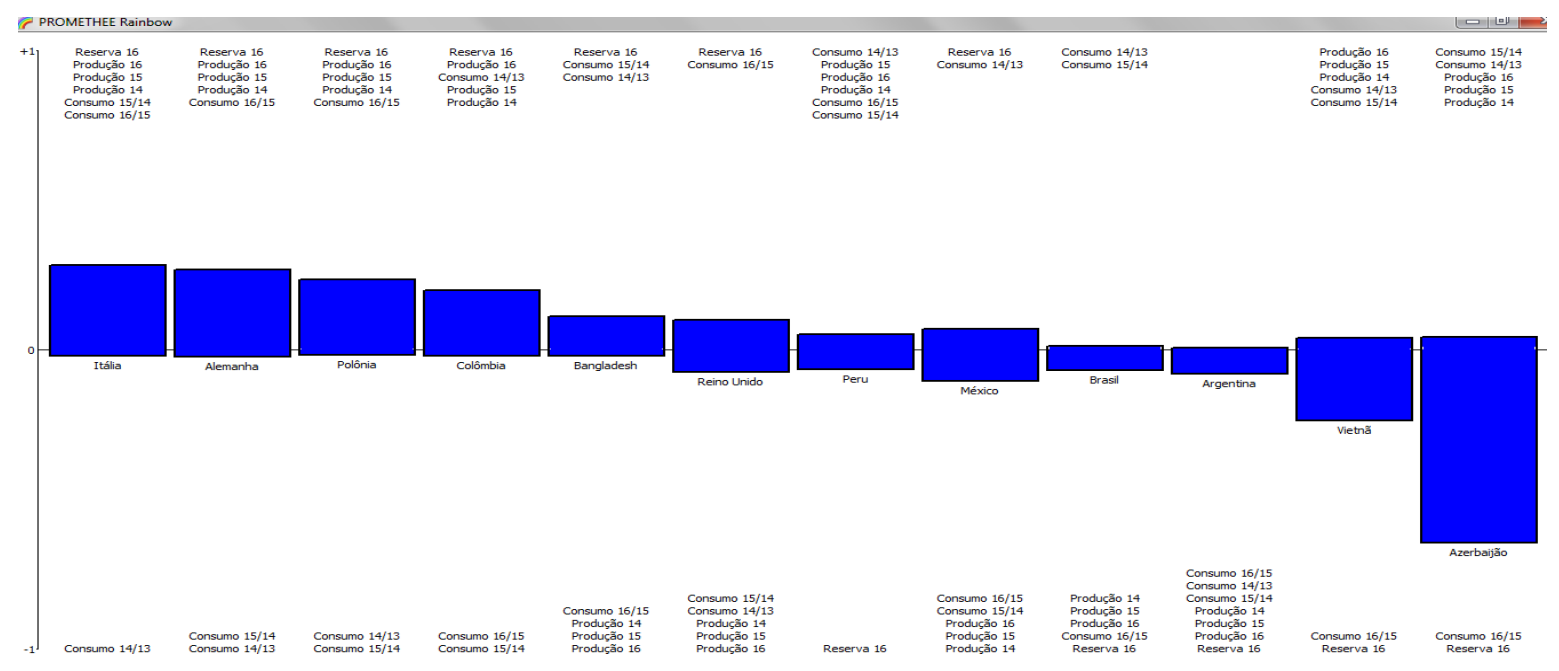


A Figura 2 relaciona todos os pontos fortes e fracos de cada país, isto é, relaciona todos os subcritérios com o melhor e o pior desempenho para cada país em comparação com os demais. Inicialmente, foi possível observar que a Itália mesmo assumindo a $1^{\text {a }}$ colocação da ordenação, não possui o melhor desempenho em todos os subcritérios. Ou seja, há países que possuem variação percentual de consumo entre 2013 e $2014\left(\right.$ SC $\left._{1}\right)$ ainda mais expressiva que a Itália, pois este subcritério é de maximização.

Além disso, também foi possível verificar que as $11^{\text {a }}$ e $12^{\text {a }}$ posições possuem os mesmos pontos fortes e fracos, no entanto destacou-se o desempenho dos subcritérios da $11^{\mathrm{a}}$ colocação.

\subsection{GRÁFICO DE REDES doS PAÍSES}

Outro gráfico gerado pelo software Visual PROMETHEE® é o gráfico PROMETHEE Network. Este gráfico pode ser fortemente utilizado a fim contribuir com o apoio à decisão, pois apresenta a posição de cada alternativa em relação às demais dentro do conjunto de alternativas estudado.

Figura 3 - Gráfico de redes dos países pelo método PROMÉTHÉE II

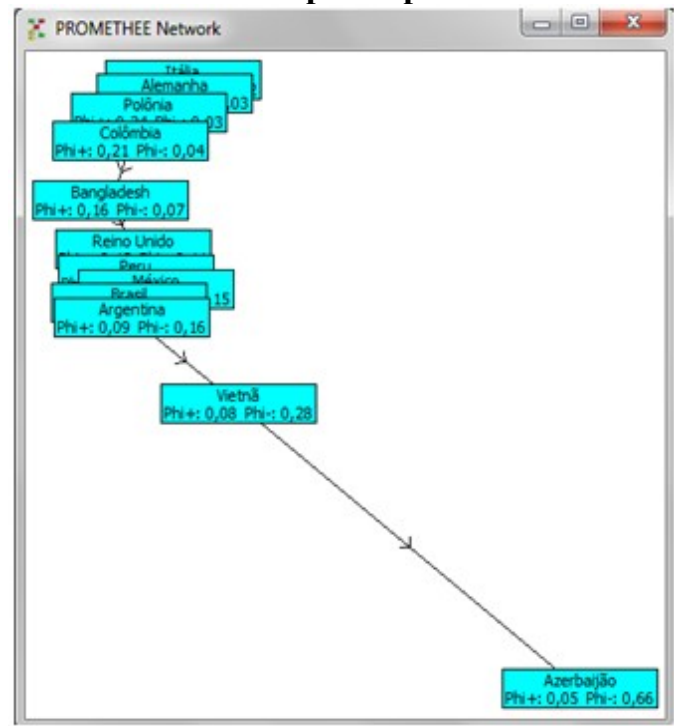

Fonte: Software Visual PROMETHEE®

Os métodos com abordagem de análise de decisão multicritério consistem em um conjunto de ferramentas que auxiliam o decisor no processo de escolha, sendo que em alguns desses é incorporado os recursos de visualização gráfica de fácil compreensão como o PROMETHEE Rankings (Figura 1) e o Gráfico de Redes (Figura 3).

O Gráfico de Redes na Figura 3 demonstrou de forma visual que apesar dos países que assumem a $1^{\mathrm{a}}, 2^{\mathrm{a}}, 3^{\mathrm{a}}, 4^{\mathrm{a}}, 5^{\mathrm{a}}$ e $6^{\mathrm{a}}$ colocações possuam o mesmo grau de atratividade II, a Itália que assumiu a $1^{\mathrm{a}}$ colocação possui uma posição de destaque em relação a estes.

Ao analisar-se as demais posições no ranking, verificou-se que a $5^{\text {a }}$ posição (Bangladesh) também assumiu posição de destaque em relação aos demais. Isto aconteceu porque os países que se encontram nas $7^{\mathrm{a}}, 8^{\mathrm{a}}, 9^{\mathrm{a}}, 10^{\mathrm{a}}$ e $11^{\mathrm{a}}$ posições possuíam o mesmo grau de atratividade de Bangladesh (Figura 1). Também foi importante notar que Reino Unido, Peru, México, Brasil e Argentina encontravam-se em posições muito próximas um do outro, diferentemente do Vietnã e do Azerbaijão.

\section{CONSIDERAÇÕES FINAIS}

Esta pesquisa apresentou uma abordagem de apoio multicritério à decisão aplicada à indústria de gás natural como contribuição ao planejamento e a tomada de decisões do Governo, agentes econômicos e demais interessados no mercado de gás natural internacional. 
Além disso, esta pesquisa demonstrou como a aplicação de uma abordagem multicritério adequada se torna uma ferramenta eficiente para um processo de análise robusta a fim de auxiliar a ordenação dos potenciais países consumidores de gás natural do mundo para o incentivo à venda deste combustível no mercado mundial.

Para estabelecer a ordenação do conjunto dos potenciais países consumidores de gás natural, foram analisados sete subcritérios quantitativos: variação de consumo de gás natural entre 2014 a 2016, produção de gás natural entre 2014 e 2016 e reservas provadas de gás natural em 2016. Todos os dados utilizados que demonstram o desempenho das alternativas estudadas nestes sete subcritérios foram obtidos a partir da publicação do Anuário Estatístico Brasileiro do Petróleo, Gás Natural e Biocombustíveis 2017 da ANP.

A abordagem multicritério constituída nesta pesquisa foi composta pelo método WINGS, utilizado para gerar os pesos de cada critério, seguido do método TOPSIS, utilizado para pré-selecionar os países que mais se aproximam de ser um potencial consumidor mundial de gás natural. Após a aplicação do método TOPSIS, foi realizada uma pré-análise de dominância entre as alternativas, a fim de excluir alternativas dominadas do conjunto de alternativas. Em seguida, foi aplicado o método PROMÉTHÉE II para estabelecer a ordenação final dos países estudados.

Com a aplicação da abordagem multicritério aqui constituída foi possível estabelecer uma ordenação dos países estudados a partir do país com maiores características em relação aos demais de ser um potencial consumidor até o que possui menos características em relação aos demais de ser um potencial consumidor de gás natural. Esta ordenação possibilitou uma forma de analisar os dados fornecidos pelo anuário da ANP de forma simultânea, uma vez que os dados de consumo, produção e reservas de gás natural de cada país são apresentados separadamente no anuário.

Também foi possível notar a partir da aplicação desta abordagem multicritério outros aspectos que podem ser destacados como fatores de contribuição ao apoio à decisão. Estes fatores foram observados a partir dos gráficos gerados pelo software Visual PROMETHEE® e trouxe sensibilidade à ordenação final dos países estudados. Assim, juntamente com a ordenação de alternativas, foi possível atribuir um grau de atratividade a cada país, conhecer a posição de cada país no conjunto de países e observar com clareza os pontos fracos e fortes de cada país em relação aos demais.

Desta forma, a abordagem multicritério aqui proposta demonstrou-se sólida no cumprimento do objetivo deste estudo. Então, abre-se um novo caminho para analisar os dados divulgados pelo anuário da ANP a fim de apoiar a decisão relacionada à ordem de prioridade ao incentivo da venda de gás natural no mercado mundial.

\section{REFERÊNCIAS BIBLIOGRÁFICAS}

Agência Natural do Petróleo, Gás Natural e Biocombustíveis [ANP]. Anuário Estatístico Brasileiro do Petróleo, Gás Natural e Biocombustíveis 2017. 2017. Disponível em: http://www.anp.gov.br/publicacoes/anuario-estatistico/3819-anuario- estatistico-2017. Acesso em: 13 set. 2018.

BANA E COSTA, C. A.; DE CORTE, J. M.; VANSNICK, J. C. On the Matemathical foundations of MACBETH. In: FIGUEIRA, J.; GRECO, S. (eds.). Multiple Criteria Decision Analysis: State of the Art Surveys. New York: Springer, v. 78, p. 409-442, 2005.

BARBA-ROMERO, S.; PROMEROL, J. C. Decisiones multicriterio: fundamentos teóricos y utilización prática. Madrid: Servicio de Publicaciones de la Universidad de Alcalá, 1997. 
BELTON, V.; STEWART, T. J. Multiple criteria decision analysis: an integrated approach. Boston: Kluwer Academic Press, p.1-5, 2002.

BILGIN, M. Geopolitics of European natural gas demand: Supplies from Russia, Caspian and the Middle East. Energy Policy, v. 37, n. 11, p. 4482-4492, 2009.

BRANS, J. P.; VINCKE, P.; MARESCHAL, B. How to select and how to rank projects: The PROMETHEE method. European Journal of Operational Research, v. 24, p. 228-238, 1986.

ENERGY INFORMATION ADMINISTRATION - EIA. International Energy Outlook 2009. DOE/EIA-0484. 2009.

ENERGY INFORMATION ADMINISTRATION - EIA. International Energy Outlook 2010. DOE/EIA-0484. 2010.

ENERGY INFORMATION ADMINISTRATION - EIA. Annual Energy Outlook 2017: with projections to 2050. 2017. Disponível em:

https:/wwww.eia.gov/outlooks/aeo/pdf/0383(2017).pdf. Acesso em: 04 set. 2018.

ENERGY INFORMATION ADMINISTRATION - EIA. Annual Energy Outlook 2018: with projections to 2050. 2018. Disponível em

https://www.eia.gov/outlooks/aeo/pdf/AEO2018.pdf. Acesso em: 13 mar. 2019.

EUROPEAN COMMISSION - EC. Second Strategic Energy Review: Securing our Energy Future. 2008.

EUROPEAN COMMISSION - EC. The January 2009 Gas Supply Disruption to the EU: An Assessment, Commission Staff Working Document Accompanying Document. 2009.

FARAMAWY, S.; ZAKI, T.; SAKR, A. Natural gas origin, composition, and processing: A review. Journal of Natural Gas Science and Engineering, v. 34, p. 34-54, June 2016.

GOMES, L. F. A. M.; ARAYA, M. C. G.; CARIGNANO, C. Tomada de decisões em cenários complexos: introdução aos métodos discretos do apoio multicritério à decisão.

São Paulo: Pioneira Thomson Learning, 2004.

GOMES, L. F. A. M.; GOMES, C. F. S. Tomada de decisão gerencial: enfoque multicritério. 5a ed. São Paulo: Atlas, 2014.

GOMES, L. F. A. M.; MARANHÃO, F. J. C. A Exploração de gás natural em Mexilhão: análise multicritério pelo método TODIM. Pesquisa Operacional, v. 28, n. $\quad 3$, p. 491509, set./dez. 2008.

HELEN, H. The EU's Energy Security Dilemma with Russia. University of Leeds. Polis Journal, v. 4, p. 1-40, winter 2010.

HWANG, C. L.; YOON, K. Multiple Attribute Decision Making: Methods and Applications - A State-of-the-Art Survey. Berlin: Springer-Verlag, 1981.

KEENEY, R. L.; RAIFFA, H. Decisions With Multiple Objectives: Preferences and Value Tradeoffs. Cambridge: Cambridge University Press, 1993.

KHOSRAVANIAN, R.; WOOD. D. A. Selection of high-rate gas well completion designs applying multicriteria decision making and hierarchy methods. Journal of Natural

Gas Science and Engineering, v. 34, p. 1004-1016, Aug. 2016.

LEÃO JR., J. C. F. Seleção de Carteira de Projetos Exploratórios em Etapas:

Agrupamento, Corte e Ordenação. Dissertação de Mestrado Profissional em Administração Rio de Janeiro, Faculdades Ibmec, Rio de Janeiro, 2007. 327p. 
MAC KINNON, M.A; BROUWER, J.; SAMUELSEN, S. The role of natural gas and its infrastructure in mitigating greenhouse gas emissions, improving regional air quality, and renewable resource integration. Progress in Energy and Combustion Science, v. 64, p. 62- 92, Jan. 2018.

MICHNIK, J. Weighted Influence Non-linear Gauge System (WINGS) - An analysis method for the systems of interrelated components. European Journal of Operational Research, v. 228, n. 3, p. 536-544, 2013.

MIETTINEN, K. Survey of methods to visualize alternatives in multiple criteria decision making problems. Operations Research Spectrum, v. 36, n. 1, p. 3-37, 2014.

MLADINEO, M; JAJAC, N.; ROGULJ, K. A simplified approach to the promethee method for priority setting in management of mine action projects. Croatian Operational Research Review, v. 7, n. 2, Prosinac, 2016.

NEVES, R. B.; PEREIRA, V.; COSTA, H. G. Auxílio multicritério à decisão aplicado ao planejamento e gestão na indústria de petróleo e gás. Production, v. 25, n. 1, p. 43- 53, jan./mar. 2015.

POMEROL, J. C.; BARBA-ROMERO, S. Multicriterion Decision in Management: Principles and Pratice. Boston/Dordrecht/London: Kluwer Academic Publishers, 2000.

RADETZKI, M. European natural gas: market forces will bring about competition in any case. Energy Policy, v. 27, n. 1, p. 17-24, 1999.

ROMERO, C. Teoría de la decisión multicriterio: conceptos, técnicas y aplicaciones. Madrid: Alianza, 1993. p. 195.

ROY, B.; BOUYSSOU, D. Aide multicritère à la decision: methods et cas. Paris: Economica, 1993.

SAATY, T. L. The Analytic Hierarchy Process. New York: McGraw-Hill International, 1980.

SANTOS, E. M.; ZAMALLOA, G. C.; VILLANUEVA, L. D.; FAGÁ, M. T. D. Gás natural: estratégias para uma energia nova no Brasil. São Paulo: Annablume, 2002. v. 1. 348p.

VINCKE, P. H. L'aide multicritère à la dècision. Bruxelles: Éditions de l'Université de Bruxelles, 1989. 179 p.

ZHONG, M.; BAZILIAN, M. D. Contours of the energy transition: Investment by international oil and gas companies in renewable energy. The Electricity Journal, v. 31, n. 1, p. 82-91, jan./feb. 2018. 